\title{
Characterizing IGBT Losses for Switched Mode Operation
}

\author{
Ronald H. Randall, Member, IEEE, Alain Laprade and Barry Wood \\ Intersil Corporation \\ 125 Crestwood Road \\ Mountaintop, PA \\ United States 18707 \\ Phone: 570-474-6761 \\ E-mail: r.h.randall@ieee.org, alaprade@intersil.com,bwood04@intersil.com
}

\begin{abstract}
Accurate loss analysis of switch mode circuits that use IGBTs as a switching device is difficult. Switching losses are a function of switch current, collector voltage and junction temperature. These three-dimensional parameters increase equation complexity and are often time consuming in their determination. However, computer programs such as Mathcad ${ }^{T M}$, Matlab ${ }^{\text {TM }}$, Excel $^{T M}$, and Table Curve ${ }^{T M}$ provide practical means of curve fitting empirical data. This paper outlines a methodology for recording and converting empirical IGBT performance data into accurate and useful equations. These equations may then be utilized as simple function calls for loss analysis calculations. The examples covered in this paper are the IGBT clamped inductive turn-off energy and $V_{C E(S A T)}$ on-state drop. The losses predicted by the empirical equations are compared with in-circuit measured losses. IGBTs representing two different technologies are modeled.
\end{abstract}

\section{Introduction}

The value of mathematical loss models for predicting power semiconductor losses operating in the switched mode is apparent in [1]-[5]. In previous work [2]-[5], switching device characteristics were modeled utilizing rough approximations based on minimal device data. The development of more accurate modeling equations for parameters such as the high voltage MOSFET $R_{d s o n}$ as a function of current and temperature [2] or the turn-off loss of IGBTs as a function of current, temperature and clamp voltage [1] is challenging at best.

The availability of software such as Mathcad, Matlab, Excel and Table Curve provide a practical means of greatly improving the model accuracy while reducing the computation drudgery. Accuracy of the model is naturally of paramount importance. It is contingent upon the accuracy of the test data and the appropriateness of the equation form. A tertiary factor is the impact of the device to device and manufacturing lot to lot parametric tolerances.

While the semiconductor manufacturer can best address parameter tolerance, the model developer should keep the data accuracy in perspective. Complicating a model to improve the correlation accuracy with empirical data may add minimal value if the parameter deviates by a much larger tolerance from device to device.
IGBT inductive switched turn-off energy loss $E_{\text {off }}$ and conduction drop $\mathrm{V}_{\mathrm{CE}(\mathrm{SAT})}$ are used as examples. Turn-off energy loss is a complex function of collector current, junction temperature and clamp voltage. A matrix of $E_{\text {off }}$ loss in $\mu$ joules and $V_{\mathrm{CE}(\mathrm{SAT})}$ conduction drop versus collector current, junction temperature and collector clamp voltage was recorded using a single pulse inductive switched test fixture Fig. 1.

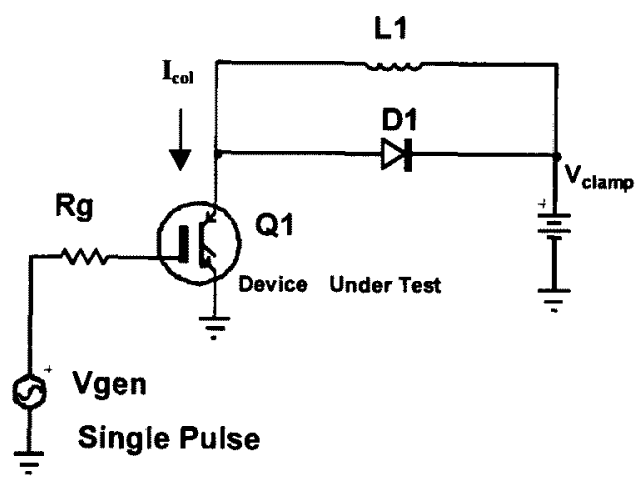

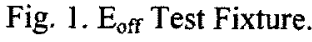

\section{Part Selection}

When a manufacturer characterizes an IGBT, several hundred parts are obtained from several production lots. On-state voltage $\left(\mathrm{V}_{\mathrm{CE}(\mathrm{SAT})}\right)$, turn-off energy $\left(\mathrm{E}_{\mathrm{off}}\right)$, and other parameters are measured. Several parts with average $\mathrm{V}_{\mathrm{CE}(\mathrm{SAT})}$ are selected. From these parts, turn-off energy is examined. A single part with close to average $E_{\text {off }}$ is selected to make the model. Because many IGBT parameters are interrelated, there will not be a single part having average $E_{\text {on }}, E_{\text {off }}$ and $V_{C E(S A T)}$. We have chosen to use the methods herein described by characterizing IGBTs HGTP12N60B3 (GEN III) [6] and HGTP12N60A4 (GEN IV) [7].

A concern in IGBT characterization is the interaction of parameters. Typically, devices with lower collectoremitter saturation voltages will have higher turn-off switching losses. This is illustrated by the GEN IV tradeoff curve in Fig. 2. The conundrum is if a model is worst case characterized for all parameters, it will result in a design based on a device that will never exist. The approach taken was to identify a device representing typical performance based on manufacturing distribution. 
A turn-off energy versus $V_{C E(S A T)}$ trade-off curve is shown in Fig. 2. A $V_{C E(S A T)}$ distribution curve based on 5000 parts is also shown.

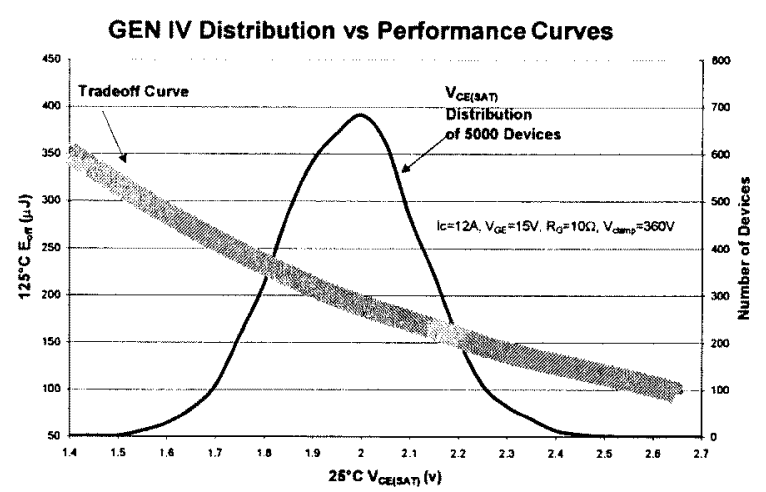

Figure 2: $E_{\text {off }}$ versus $V_{C E(S A T)}$ trade-off curve

\section{Recording Data}

The empirical turn-off energy and conduction drop data were recorded using the test circuit Fig. 1. This circuit closely represents the inductive turn-off and hard recovery turn-on conditions of typical power circuits such as the Boost power factor correction (PFC) circuit.

The test circuit, Fig. 1, is designed such that diode D1 and the IGBT under test may be preheated to a specific temperature prior to initiating a single test pulse. The clamp voltage $\mathrm{V}_{\text {clamp }}$ is variable such that the device under test (DUT) may be switched at specific junction temperatures and voltages. The magnitude of the inductor L1 was varied so that varying switching currents could be obtained for constant DUT conduction times.

Turn-off loss measurements were made using a single pulse with the device preheated to the specific junction temperature. The energy loss per pulse is recorded as the integral of the total switching energy pulse including collector current tailing. Reference the $E_{\text {off }}$ trace in Fig. 3.

If the specific application is one in which the IGBT operates at a constant clamp voltage, then the curve fit task may be greatly simplified. The boost PFC circuit is a good example of an application where the IGBT performance need only be evaluated at a single clamp voltage. That is, each time the device is switched off, it is clamped to the PFC DC output voltage. However, if the IGBT will be turned off under varying clamp voltages or a ubiquitous model is desired, then the $V_{\text {clamp }}$ impact on turn-off loss must be considered and the equation model becomes more complex. In the following analysis, it was assumed that the turn-off clamp voltage is a variable parameter and $E_{\text {off }}$ energy measurements were made at four clamp voltages (i.e. 100, 200, 300, 400V).

\section{Determining Equation Form}

The success of a model fit is contingent upon the appropriateness of the equation form used. Relating the equation form to the physical characteristics of the device and the circuit it is operating in can greatly simplify the process. The equation form may be determined by direct interface with the device designer, careful analysis of the device's time domain performance and/or trial and error.

Fig. 3 illustrates the turn-off voltage, current characteristics of the GEN IV IGBT. Three distinctive transition regions exist during the turn-off period.

$t_{1}$ - The period when the collector voltage rises rapidly to the clamp voltage.

$t_{2}$ - The period when the collector current falls rapidly.

$t_{3}$ - The current tailing period during which the collector current decays exponentially to zero.

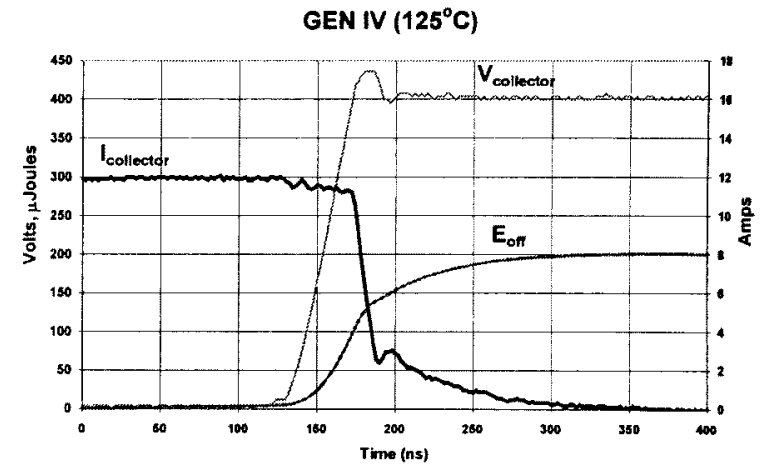

Fig. 3. IGBT turn-off current and voltage waveshapes.

The slight reduction in collector current during period $t_{1}$ is the result of a portion of the load current charging the power circuit parasitic capacitances at a nearly constant collector $\mathrm{dv} / \mathrm{dt}$

Losses associated with each segment of the turn-off period are illustrated in Fig. 4. The $E_{\text {off }}(W)$ trace is the instantaneous power loss. The second trace is the integral of power loss from the beginning of the turn-off switch period to that point in time. This second curve is important in that it provides a measure of the energy dissipated during each portion of the turn-off period. The magnitude of this second, integral, curve in Fig. 4 was used to record the total energy dissipated in the IGBT during the turn-off period. The $200 \mu$ joule value in Fig. 4 represents one data point in the turn-off curve fit equation data matrix.

Inspection of Fig. 4 reveals that an appreciable portion of the energy lost during IGBT turn-off may be attributed to the tail current. Since the tail current varies as a function of collector current and temperature, the $E_{\text {off }}$ energy dissipated during turn-off is a complex function of current, temperature and collector clamp voltage.

To determine the form of the equation required for fitting the $\operatorname{Eoff}(V, I, T j)$, multiple two-dimensional plots were made of the empirical data holding two parameters constant and plotting the remaining parameter against $E_{\text {off }} \cdot E_{\text {off }}$ was plotted versus $I$ for constant $T_{j}$ and $V_{\text {clamp }}$ 
etc. By test fitting each of these two-dimensional plots, the basic form of $E_{o f f}$ in $\mu$ joules (1) was developed.

\section{GEN IV $\left(125^{\circ} \mathrm{C}\right)$}

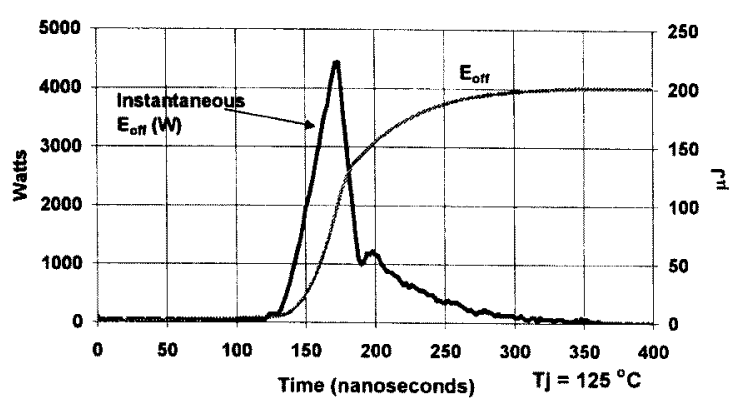

Fig. 4. $E_{\text {off }}$ watts and accumulated $\mu$ joules as $f(t)$

$$
\begin{aligned}
& \text { Eoff }\left(V_{\text {clamp }}, I, T j\right)= \\
& \frac{V_{\text {clamp }}}{400} \cdot(b 8+b 9 \cdot I) \cdot\left[\begin{array}{l}
(b 1+b 2 \cdot T j) \cdot e^{b 3 \cdot I}+ \\
(b 4+b 5 \cdot T j) \cdot I+ \\
b 6 \cdot I^{2}+b 7 \cdot T j
\end{array}\right]
\end{aligned}
$$

\section{Optimizing the Fit}

\subsection{Turn-off Loss $\left(\mathbf{E}_{\text {off }}\right)$}

The $E_{\text {off }}$ test data was recorded into a matrix as illustrated in (2) with each row representing a test point. The TestData matrix (2) was then converted to vectors (3) such that the three independent variables $V_{\text {clamp}}, I_{\text {col }}$ and $T j$ and the dependant variable $E_{\text {off }}$ are vectors equal in length to the number of rows in (2).

$$
\text { TestData }=\left[\begin{array}{cccc}
E_{o f f_{p 1}} & V_{\text {clamp }_{p 1}} & I_{c o l_{p 1}} & T j_{p 1} \\
E_{o f f_{p 2}} & V_{\text {clamp }_{p 2}} & I_{\text {col }_{p 2}} & T j_{p 2} \\
\vdots & \vdots & \vdots & \vdots \\
E_{o f f_{p m}} & V_{\text {clamp }_{p m}} & I_{\text {col }_{p m}} & T j_{p m}
\end{array}\right]
$$

$$
\begin{aligned}
& \overrightarrow{E o f f}_{p}=\text { TestData }_{p, 0} \\
& \overrightarrow{V C}=\text { TestData }_{p, 1} \\
& \vec{I}_{p}=\text { TestData }_{p, 2} \\
& \overrightarrow{T j}_{p}=\text { TestData }_{p, 3}
\end{aligned}
$$

where,

$$
p=[0 \ldots r o w s(\text { TestData })-1]
$$

The length of data vectors $\overrightarrow{E_{\text {off }}}, \overrightarrow{V C}, \vec{I}$ and $\overrightarrow{T_{j}}$ in (3) are equal to the total number of empirical test conditions. If $\mathrm{E}_{\text {off }}$ is measured for $n 1$ collector voltages, $n 2$ collector currents and $n 3$ junction temperatures, each of the data vectors will contain $n 1 \cdot n 2 \cdot n 3$ data points. Tests were performed for eleven currents, four clamp voltages and five junction temperatures resulting in 220 empirical data points.

Equation (5) is a modification of (1) with coefficients b1 ...b9 defined as variables for the error minimization process. Equation $(6) f(b 1, b 2 \ldots b 9)$ represents the error squared function to be minimized for a least squares curve fit solution. This function describes the sum of the curve fit errors squared. Equation (7) determines the $\overrightarrow{E_{\text {off }}}$ loss coefficients that minimize the error function in (6) by utilizing Mathcad's Minimize function.

$$
\begin{aligned}
& \operatorname{Eoff}\left(V_{\text {clamp }}, I_{i}, T j_{i}, b 1, b 2, \ldots, b 9\right)= \\
& \frac{V_{\text {clamp }}}{400} \cdot(b 8+b 9 \cdot I) \cdot\left[\begin{array}{l}
\left(b 1+b 2 \cdot T j_{i}\right) \cdot e^{b 3 \cdot I_{i}}+ \\
\left(b 4+b 5 \cdot T j_{i}\right) \cdot I_{i}+ \\
b 6 \cdot I_{i}{ }^{2}+b 7 \cdot T j_{i}
\end{array}\right]
\end{aligned}
$$

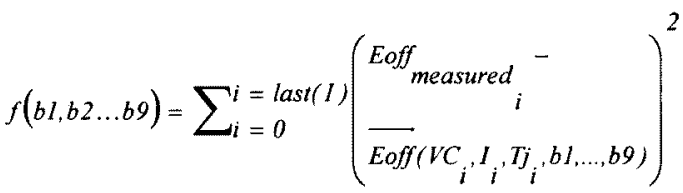$$
\left[\begin{array}{c}
b I \\
b 2 \\
\vdots \\
b 9
\end{array}\right]=\operatorname{Minimize}(f, b 1, b 2 \ldots b 9)
$$

While Mathcad's linfit and genfit curve fit functions provide an automated method, the Minimize function provides a means of simultaneous fitting four variables. The four variables are $\overrightarrow{E_{\text {off }}}, \overrightarrow{V C}, \vec{I}$ and $\overrightarrow{T_{j}}$. The Minimize function also provides a means of weighting the data by inserting multipliers into the least squares (6). This allows heavier weighting in the areas of most concern. Heavier weighting of the currents in the specific application operating area may improve the analysis accuracy.

Curve fit weighting may also be achieved with additional test data points in the areas of primary concern. For example, if the primary operation of the circuit is within the range of 300 to 400 volts and the junction temperature is anticipated to be in the 100 to $125^{\circ} \mathrm{C}$ range, then the major portion of the test data should be concentrated within these ranges. The Minimize function (7) will then automatically optimize the fit in the desired area. Inserting data outside the normal operating range but within component rating will insure that the equations 
do not diverge outside of the operating range. It is good practice to test plot the equations to determine their stability outside the normal range. It should also be understood that applying the equations outside of their measured range may result in erroneous values.

The division by 400 in (5) normalizes $V_{\text {clamp }}$ with respect to the maximum value. This increases the values of coefficients bl through b9. It has been observed that if this is not done, numerical convergence problem may occur. When using the Minimize function, a seed value is required as a first approximation for coefficients b1 through b9. Because of the large number of unknown variables in the expression, it may be necessary to reinitialize the seed values a few times until an optimal answer is obtained. It is believed that the numerical algorithms used in the math programs may be encountering multiple saddle points, forcing a recalculation to confirm an optimal answer. The accuracy of the equation fit is easily monitored by observing the value generated by the least squares function (6).

GEN III and GEN IV IGBTs were tested for eleven current measurements, at four clamp voltages and five junction temperatures. Results are plotted versus test data in Figs. 6 and 7. Symbols represent tested data. Traces represent results from (1).

The GEN III tests were performed using $V_{G}=15 \mathrm{~V}$ and $\mathrm{R}_{\mathrm{g}}=25 \Omega$. An $\mathrm{R}_{\mathrm{g}}=10 \Omega$ and $\mathrm{V}_{\mathrm{G}}=15 \mathrm{~V}$ were used for the GEN IV.

Table 1 lists the coefficients for b1 through b9 for the IGBT types characterized. These values were used for generating the traces in Figs. 6 and 7.

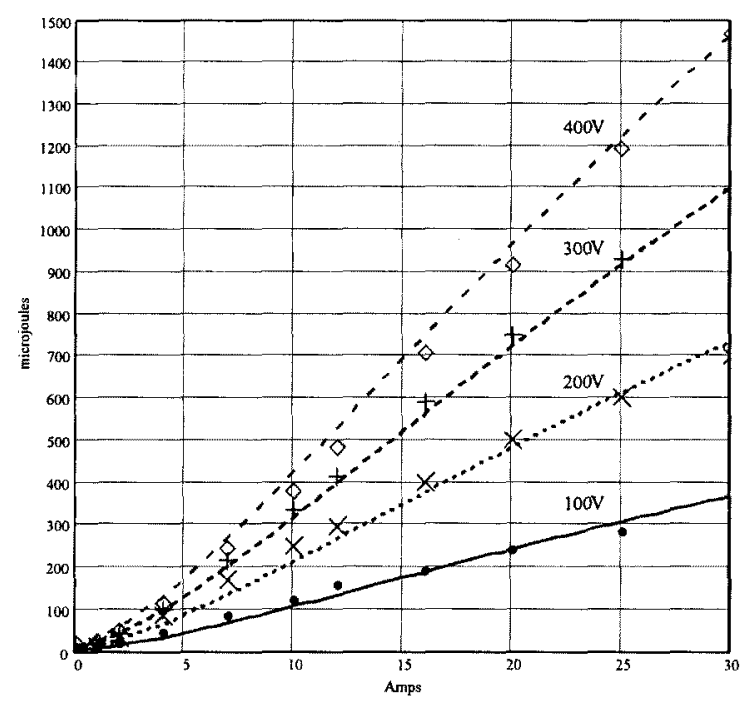

Fig. 6. GEN III $E_{\text {off }}$ characteristic $\left(100^{\circ} \mathrm{C}\right)$ :

- Symbols represent measured data

- Traces represent calculated data

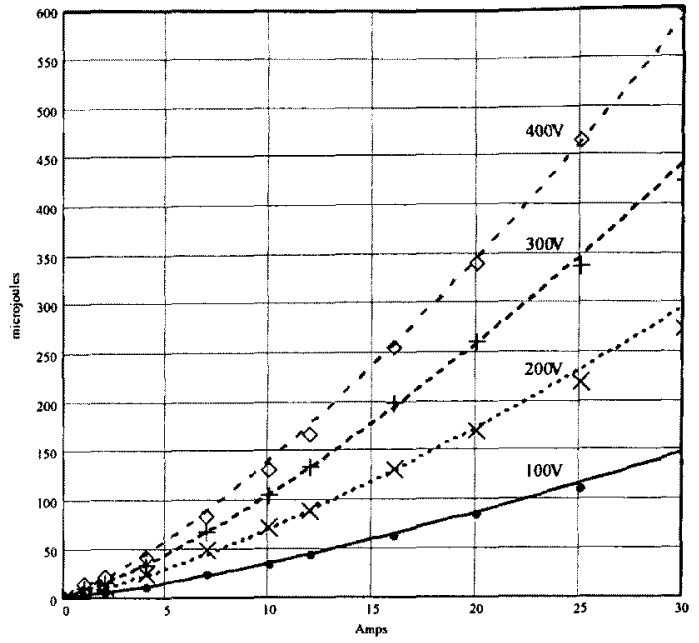

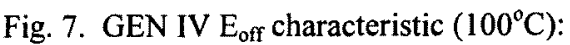

- Symbols represent measured data

- Traces represent calculated data

Table 1: $E_{\text {off }}$ equation coefficients

\begin{tabular}{||l|l|l||}
\hline & \multicolumn{1}{|c|}{ GEN III } & \multicolumn{1}{c|}{ GEN IV } \\
\hline b1 & 0.6140 & $7.393 \times 10^{-2}$ \\
\hline b2 & -1.239 & $1.229 \times 10^{-2}$ \\
\hline b3 & -0.2567 & -0.2266 \\
\hline b4 & 8.464 & $8.534 \times 10^{-2}$ \\
\hline b5 & 0.3255 & $4.472 \times 10^{-3}$ \\
\hline b6 & 0.5398 & $6.298 \times 10^{-3}$ \\
\hline b7 & -1.1299 & $-1.224 \times 10^{-2}$ \\
\hline b8 & 1.3006 & 28.89 \\
\hline b9 & $-1.293 \times 10^{-2}$ & $5.043 \times 10^{-4}$ \\
\hline
\end{tabular}

\subsection{Conduction Drop ( $\left.\mathrm{V}_{\mathrm{CE}(\mathrm{SAT})}\right)$}

Repeating the techniques from 5.1, parameters for the coefficients of the $V_{C E(S A T)}$ expression may be determined for (8). Equation (8) coefficients al through all for the GEN III and GEN IV IGBTs are tabulated in Table 2.

$$
V f_{l g b t}(I, T j)=\left[\begin{array}{l}
\left(a I \cdot T j^{2}+a 2 \cdot T j+a 3\right) \cdot e^{a l 0 \cdot I}+ \\
\left(a 4 \cdot T j^{2}+a 5 \cdot T j+a 6\right) \cdot I^{a I I}+ \\
\left(a 7 \cdot T j^{2}+a 8 \cdot T j+a 9\right)
\end{array}\right]
$$

The form of (8) is an exponential term that shapes to the forward drop of low current values, an $\mathrm{I}^{\text {all }}$ term that shapes the forward drop at higher currents and a constant term that sets the offset threshold voltage. The second order temperature terms in front of each of the current terms serves to adjust the individual component weighting as a function of the IGBT junction temperature.

GEN III and GEN IV IGBTs were tested to this algorithm. Results are plotted versus test data in Figs. 8 
and 9. Symbols represent tested data. Traces represent results from (8).

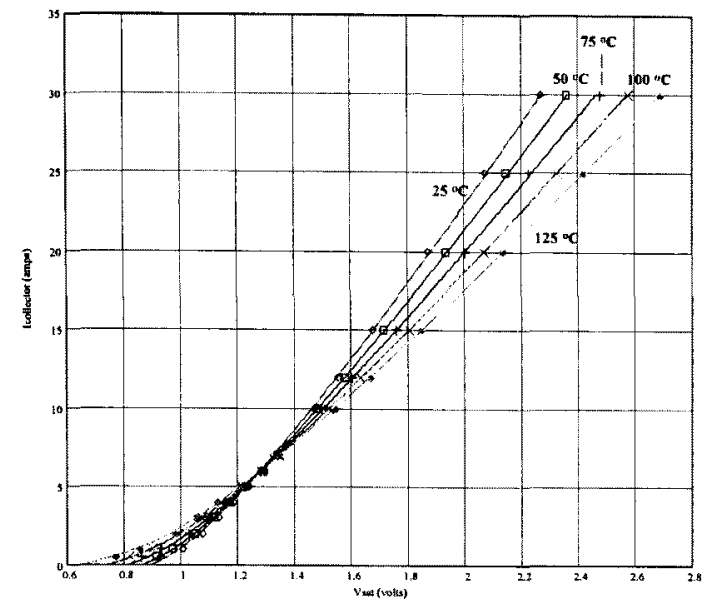

Fig. 8. GEN III $\mathrm{V}_{\mathrm{CE}(\mathrm{SAT})}$ characteristic - Symbols represent measured data

- Traces represent calculated data

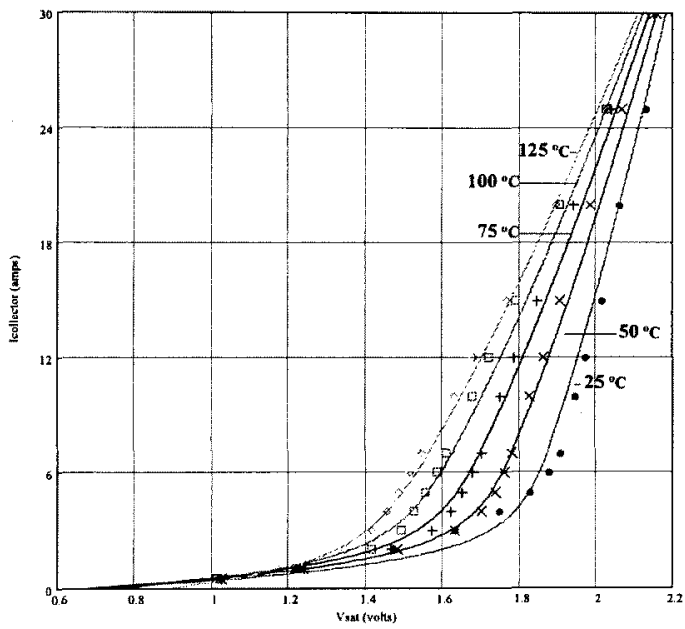

Fig. 9. GEN IV $V_{\mathrm{CE}(\mathrm{SAT})}$ characteristic

- Symbols represent measured data

- Traces represent calculated data

Table 2: $V_{C E(S A T)}$ equation coefficients

\begin{tabular}{|l|l|l||}
\hline & \multicolumn{1}{|c|}{ GEN III } & \multicolumn{1}{c|}{ GEN IV } \\
\hline a1 & $-1.265 \times 10^{-5}$ & $3.000 \times 10^{-5}$ \\
\hline a2 & $8.658 \times 10^{-4}$ & $1.758 \times 10^{-3}$ \\
\hline a3 & $-5.668 \times 10^{-2}$ & -1.088 \\
\hline a4 & $6.277 \times 10^{-7}$ & $-6.348 \times 10^{-7}$ \\
\hline a5 & $2.187 \times 10^{-4}$ & $3.788 \times 10^{-4}$ \\
\hline a6 & $6.792 \times 10^{-2}$ & $2.763 \times 10^{-2}$ \\
\hline a7 & $-4.717 \times 10^{-6}$ & $1.126 \times 10^{-5}$ \\
\hline a8 & $-7.228 \times 10^{-4}$ & $-6.016 \times 10^{-3}$ \\
\hline a9 & 0.9574 & 1.860 \\
\hline a10 & -0.9500 & -0.8650 \\
\hline a11 & 0.8500 & 0.7470 \\
\hline
\end{tabular}

\section{Application}

The development of accurate equations for calculating IGBT $E_{\text {off, }} E_{\text {on }}$ and $V_{C E(S A T)}$ provide a powerful set of design analysis tools. These equations may be used as an electronic datasheet providing detailed device performance at the specific points of interest or incorporated into the overall design equations. The value of these equations is apparent in [1]-[5].

For example, if expressions are developed for the IGBT turn-on and turn-off currents in a specific application, the IGBT losses may be calculated as in (9), (10) and (11).

$$
\begin{gathered}
\text { IgbtTurnOnWatts }\left(V_{\text {clamp }}, I_{\text {ton }}, T j\right)= \\
f_{s} \cdot \text { Eon }_{\text {lgbt }}\left(V_{\text {clamp }}, I_{\text {ton }}, T j\right) \\
\text { IgbtTurnOffWatts }\left(V_{\text {clamp }}, I_{\text {toff }}, T j\right)= \\
f_{s} \cdot \text { Eoff }_{\text {igbt }}\left(V_{\text {clamp }}, I_{\text {toff }}, T j\right) \\
\text { IgbtOnStateWatts }(I, T j)=D F \cdot I_{o n} \cdot V f_{\text {igbt }}\left(I_{o n}, T j\right)
\end{gathered}
$$

where:

$f_{s}$ is the IGBT switching frequency

$D F$ is the IGBT conduction duty factor

$I_{\text {Ion }}$ is the IGBT current at turn-on

$I_{\text {toff }}$ is the IGBT current at turn-off

$I_{o n}$ is the average current conduction during on-time

$V$ is the IGBT time variable voltage

$I \quad$ is the IGBT time variable current

The versatility of these equations is illustrated in [1] where they are used to predict the losses in an IGBT operating in a $600 \mathrm{~W}$ PFC circuit. In [1], a correlation of 23.8 watts measured versus 25.2 watts predicted was achieved. Further, because the impact of junction temperature on IGBT losses is imbedded in the equations, the IGBT operating junction temperature may be determined by introducing the device cooling constants into equation (12) and performing multiple iterations. Successive calculations are made until convergence is obtained (Fig. 10). Computation time is of the order seconds.

$$
\begin{aligned}
& T_{j_{n}}= \\
& I G B T \_ \text {TotalLosses }\left(V_{\text {clamp }}, I_{\text {ton }}, I_{o n}, I_{\text {toff }}, T_{j_{(n-1)}}\right) \cdot R_{\theta J A} \\
& +T_{\text {amb }}
\end{aligned}
$$

where,

$R_{\theta J A}$ is the junction to ambient thermal impedance

$T_{a m b}$ is the ambient temperature 
The versatility of implementing IGBT loss functions (1) and (8) becomes apparent when losses (9), (10) and (11) are summed and plotted as a function of a variable as in Fig. 11. Fig. 11 illustrates the total losses in an IGBT operating in a $600 \mathrm{~W}$ PFC circuit.

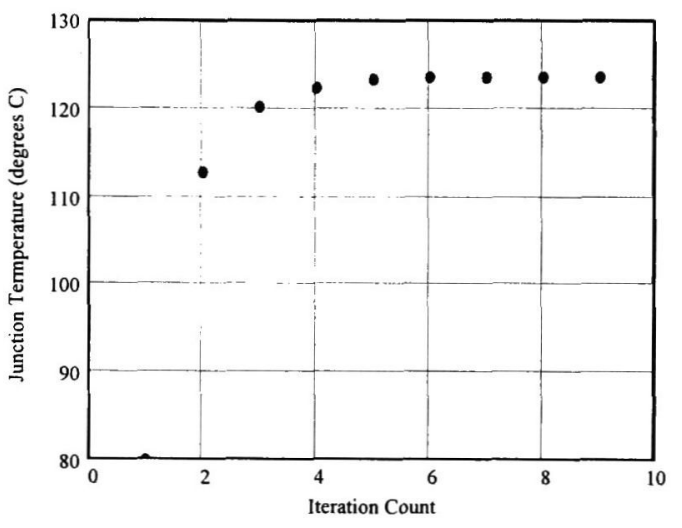

Fig. 10. Iterative junction temperature calculation

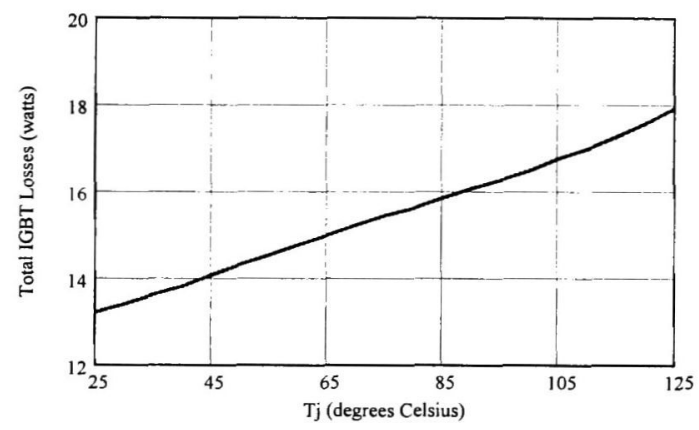

Figure 11: Total losses versus junction temperature

\section{Conclusion}

A logical approach for generating accurate IGBT performance equations from empirical characterization data has been described. The approach takes advantage of the multiple variable curve fitting capabilities of Mathcad. The approach is not limited to Mathcad and may be implemented using other mathematical analysis programs such as Excel and Matlab. The methodology is validated using two IGBT types that represent different silicon technologies.

The developed equations are valuable in that they provide a means of predicting IGBT losses as a function of temperature, voltage and current. By applying the developed equations to specific applications, it is possible to predict device operating temperatures and losses.

(Manuscript received March 15, 2000)

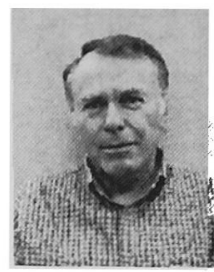

Ronald H. Randall (Member IEEE since 1965) received the BS in Electrical Engineering from Tri-State University, Angola, Indiana in 1965. He has worked in power electronics design and development for 35 years and holds 5 patents in the power electronics field. He has been engaged with Intersil Corporation as a Contract Engineer since 1997. At Intersil his efforts have been in the characterization and application of IGBTs in SMPS applications. His main interests are in the development of simulation and modeling techniques for power electronic circuits.
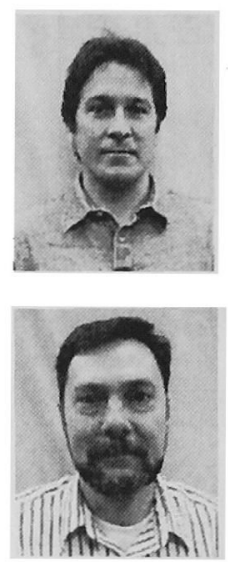

Alain Laprade obtained a B.Eng. in 1983 and a M. Eng. in 1987 at McGill University in Montreal, Canada. He has a worked as a power supply designer for 14 years in applications including computer power, high power telecommunications and space designs. He has been with Intersil Corporation since February 1998. His title is Team Leader with the Cellular Phone Group.

Barry Wood received his engineering degree from Wilkes University in 1993. He is a Senior Design Engineer with Intersil Corp. For the last six years, he has worked in characterization.

\section{References}

[1] Laprade, A., Bober, G., Randall, R. H. (1999), "Numerical method for evaluating current, voltage and temperature dependant IGBT switching and conduction losses, "PCIM Europe 1999, pp. 329-334, June 1999.

[2] Kolar, J.W., Ertl, H., and Zach, F.C. (1998), "How to include the dependency of the $\mathrm{R}_{\mathrm{ds}(\mathrm{on})}$ of power MOSFET's on the instantaneous value of the drain current into the calculation of the conduction losses of high-frequency three-phase PWM inverters", IEEE Trans. Ind. Electronics, Vol. 45, No.3, pp. 369-375, June 1998.

[3] Masserant, B. and Stuart, T.A., (1996), "Experimental verification of calculated IGBT losses in PFCs" ,IEEE Transactions on Aerospace and Electronic Systems, Vol. 32, No. 3, pp. 1154-1158, July 1996.

[4] Stuart, T.A., and Shaoyan Ye (1995), "Computer simulation of IGBT losses in PFC circuits", IEEE Transactions on Aerospace and Electronic Systems, Vol. 31, No. 3, pp. 1167-1173, July 1995.

[5] Stuart, T.A., and Shaoyan Ye (1994), "Computer simulation of IGBT losses in PFC circuits", IEEE $4^{\text {th }}$ Workshop on Computers in Power Electronics, pp.85-90, 1994.

[6] Intersil Corporation, Mountaintop, PA, Data Sheet HGTP12N60B3, File Number 4410.2, 2000.

[7] Intersil Corporation, Mountaintop, PA, Data Sheet HGTP12N60A4, File Number 4656.2, 1999 . 